\title{
Flight Evaluation of an LPV Sliding Mode Observer for Sensor FTC
}

\author{
Lejun Chen, Halim Alwi, Christopher Edwards and Masayuki Sato
}

\begin{abstract}
This paper develops a sliding mode sensor fault tolerant control scheme for a class of LPV systems. It incorporates a sliding mode observer which reconstructs the unknown sensor faults based on only the system inputs and outputs. The reconstructed sensor faults are used to compensate the corrupted sensor measurements before they are used in the feedback controller. Provided accurate fault estimates can be computed, near nominal control performance can be retained without any controller reconfiguration. Furthermore, the closed-loop stability of the FTC scheme, involving both a sliding mode controller and a sliding mode observer, is rigorously analysed. The proposed scheme is validated using the Japan Aerospace Exploration Agency's Multi-Purpose Aviation Laboratory (MuPAL- $\alpha$ ) research aircraft. These flight tests represent the first validation tests of a sliding mode sensor FTC scheme on a full scale aircraft.
\end{abstract}

Keyword: sliding mode control, fault tolerant control, fault detection and diagnosis, flight test.

\section{INTRODUCTION}

D ESPITE all the research interest in fault detection and diagnosis (FDD) and fault tolerant control (FTC) [1], there exists a significant theory/practice gap. In recent years, a series of European funded projects have sought to develop a range of model based approaches in an effort to narrow this gap [2]. As argued in [26], although the signal-based FDD methods do not require the knowledge of models, the design parameters could be insensitive to the models. In addition, under poor excitation, the issues corresponding to model observability and parameter identifiability need to be addressed. Driven by the aerospace industry interest, in this paper, a model based approach is adopted. In most application areas - often because of their safety critical nature - new model based FDD and FTC are only tested by simulations. This is particularly true in the aerospace sector: literature describing the testing of model based estimation schemes in-flight, or on full scale piloted aircraft (as opposite to UAVs), is limited. Recent European projects validated a range of model based FDD schemes at a system integration level using Airbus's actuator 'integration bench' and the 'Iron-bird' platform [3]. The methods tested included sliding mode methods [11], a geometric design [12], $\mathcal{H}_{-} / \mathcal{H}_{\infty}$ filters [6], and a physical model based approach [7]. In the follow-up project (RECONFIGURE), integrated

Lejun Chen is with School of Aerospace, Transport and Manufacturing, Cranfield University, UK (e-mail: Lejun.Chen@cranfield.ac.uk).

Halim Alwi and Christopher Edwards are with College of Engineering, Mathematics \& Physical Sciences, University of Exeter, UK (e-mail: C.Edwards@exeter.ac.uk, h.alwi@exeter.ac.uk).

Masayuki Sato is with Japan Aerospace Exploration Agency, Mitaka, Tokyo 181-0015, Japan (e-mail: sato.masayuki@jaxa.jp).
FTC/FDD approaches were validated and demonstrated using the Airbus desktop simulator [4].

Despite the advancements in Technology Readiness Levels (TRLs) achieved during the European projects ADDSAFE and RECONFIGURE, only one FDD scheme, based on an extended Kalman filter formulation, was flight tested and certified on the A350-XWB aircraft [18]. Such a validation is usually considered the final 'top level' check in the aviation industrial validation process. The recent H2020/Japan co-funded project 'Validation of Integrated Safety-enhanced Intelligent flight cONtrol' (VISION) sought to validate FDI schemes via flight tests on the Japan Aerospace Exploration Agency (JAXA)'s Multipurpose Aviation Laboratory (MuPAL$\alpha$ ) aircraft [8], [21]. Sliding mode, $\mathcal{H}_{\infty}$ and adaptive estimation schemes have been developed within the VISION project, and some initial results were reported in [5], [9], [10]. In [5], an actuator FTC scheme involving a state feedback sliding mode controller was flight validated under the assumption that all sensors were assumed to be fault free.

It is well known that sliding mode observers (SMOs) have the capability to track plant measurements whilst simultaneously reconstructing the internal unmeasurable signals including the system states [14]. This unique property distinguishes SMOs from other types of observers, such as high gain observers [15] and $\mathcal{H}_{\infty}$ fault detection filters [16]. Another important property of SMOs is they can provide finite time convergence of the state estimation errors to zero. Once the sliding mode is attained, the so-called 'equivalent output error injection' signal, required to maintain sliding [14], can be used to reconstruct the unknown signals (in this case faults) in the FDD problem. In a sensor fault scenario, if they are well reconstructed by the SMO, a 'virtual sensor' can be created by correcting the corrupted measurement using the fault estimate [13]. Using the virtual sensor as part of the feedback loop (rather than the corrupted measurement) helps retain close to nominal fault free closed-loop performance in the presence of faults, without reconfiguring the underlying baseline control law [11]. In recent years several SMO based FTC schemes have been developed. In [23], [24], the perturbations and the faults were identified using a higher order sliding mode (HOSM) differentiator and a dynamic sliding surface was developed to deal with unmatched perturbations. The work in [25] describes a strategy based on a combination of a feedback linearisation and a quasi-continuous HOSM controller.

In this paper, a sensor FTC scheme based on an SMO is developed and flight tested on JAXA's MuPAL- $\alpha$ aircraft. The first order SMO used in this paper follows the general formulation of [22], [19]. However, the work in [22] mitigates 
the effects of erroneous scheduling parameters through an adaptation scheme, whilst the work in [19] considers the simultaneous optimization of the SMO gains and the $\mathcal{H}_{\infty}$ controller gains. The flight tests focus on the lateral-directional dynamics, and various yaw rate sensor fault scenarios are considered. A yaw rate sensor fault is critical since it leads to non-trim offsets of the control surfaces and a non-optimised aerodynamic configuration. It also represents one of the scenarios needed to be certified by the aviation industry [3].

The main contribution of this paper is it describes, as far as the authors are aware, the first flight validations of an SMO based FTC scheme on a piloted aircraft in the presence of sensor faults. Compared with [5], the emphasis of this flight validation was on the observer performance in the face of sensor faults. Furthermore from theoretical point of view, in this paper, the closed-loop stability of the FTC scheme, including both a sliding mode observer component and a sliding mode control component, is rigorously investigated. In [5], no observer is employed (and hence there is no controller/observer stability analysis) but instead the robustness of the sliding mode controllers are exploited in an actuator fault scenario.

\section{VISION AND FAULT SCENARIOS}

The H2020/Japan co-funded project VISION seeks to develop and validate in-flight FDD/FTC techniques for aircraft guidance, navigation and control, with the key objective of increasing TRLs. The proposed architecture of the FTC scheme developed and discussed in this paper is a simple one (see Fig. 1).

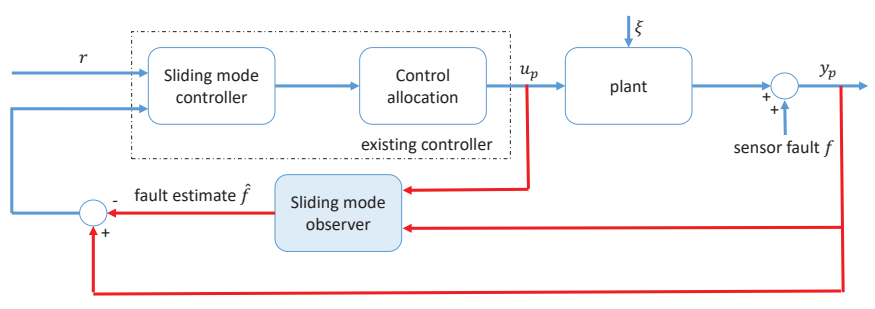

Fig. 1. The scheme of sensor FTC using SMO

In Fig. 1, an SMO reconstructs the unknown sensor fault $f$, based only on the inputs $u_{p}$ and outputs $y_{p}$. The reconstructed sensor faults $\hat{f}$ are then used to compensate the corrupted sensor measurements before they are fed back to the controller such that near nominal control performance can be retained in the face of the sensor faults, without the need of reconfiguring the controller. The augmented closed loop system in Fig. 1 must be analysed taking into account the observer dynamics. This is the main theoretical contribution of this paper.

\section{LPV SLIDING MODE OBSERVER DESIGN}

Consider an uncertain LPV system affected by sensor faults

$$
\begin{aligned}
\dot{x}_{p}(t) & =A_{p}(\rho) x_{p}(t)+B_{p}(\rho) u_{p}(t)+M_{p}(\rho) \xi\left(x_{p}, t\right) \\
y_{p}(t) & =x_{p}(t)+H_{p} f(t)
\end{aligned}
$$

where, $A_{p}(\rho) \in \mathbb{R}^{n \times n}, B_{p}(\rho) \in \mathbb{R}^{n \times m}, M_{p}(\rho) \in \mathbb{R}^{n \times k}$ and $H_{p} \in \mathbb{R}^{n \times q}$ where $q<n$. Here $H_{p}$ represents the sensor fault distribution matrix and it is assumed to be rank $q$. Furthermore the columns of $H_{p}$ are assumed to belong to the standard basis for $\mathbb{R}^{n}$. In (1) it is assumed that $u_{p}(t)$ and $y_{p}(t)$ are measurable, and that $y_{p}(t)$ represents the measured state $x_{p}(t)$ (potentially corrupted by the sensor faults represented by $f(t)$ ). The signal $f(t)$ is assumed to be piecewise differentiable and unknown, and subject to $\|f(t)\| \leq \alpha$. Finally, the signal $\xi\left(x_{p}, t\right)$ denotes lumped (possibly unmatched) system uncertainty which is assumed to be bounded according to $\|\xi(\cdot)\| \leq c_{1}\left\|x_{p}(t)\right\|+c_{2}(t)$ where $c_{1}$ is a known positive fixed scalar and the function $c_{2}(t)$ is unknown but is subject to $\left\|c_{2}(t)\right\| \leq d$ where $d$ is a known positive scalar. In this paper, it is assumed the scheduling parameters $\rho \in \Omega \subset \mathbb{R}^{r}$ are perfectly measurable and belong to a compact set $\Omega$. Furthermore, all the system matrices $A_{p}(\rho), B_{p}(\rho)$ and $M_{p}(\rho)$ in (1) are assumed to be affinely dependent on $\rho$.

Since by assumption $H_{p}$ is composed of columns from the standard basis for $\mathbb{R}^{n}$, by permutating the components of $y_{p}$ and partitioning, it is easy to obtain the form

$$
\left[\begin{array}{l}
y_{p, 1}(t) \\
y_{p, 2}(t)
\end{array}\right]=\left[\begin{array}{l}
C_{1} \\
C_{2}
\end{array}\right] x_{p}(t)+\left[\begin{array}{c}
0 \\
I_{q}
\end{array}\right] f(t)
$$

where $C_{1} \in \mathbb{R}^{(n-q) \times n}$ has full row rank and $C_{2} \in \mathbb{R}^{q \times n}$. In (2), $y_{p, 2}(t)$ denotes the outputs which are potentially corrupted by sensor faults whereas the subset $y_{p, 1}(t)$ are fault free. The observer proposed in [19] will now be described to estimate the faults $f(t)$. As in [14], define a (stable) filter in the form

$$
\dot{z}_{f}(t)=-A_{f} z_{f}(t)+A_{f} y_{p, 2}(t)
$$

where $z_{f}(t) \in \mathbb{R}^{q}$ and the matrix $A_{f}$ is symmetric positive definite (s.p.d). From (1) and (3) create an augmented system with state vector $\left[\begin{array}{cc}x_{p}^{T} & z_{f}^{T}\end{array}\right]^{T}$. Exploiting the fact that the rows of $C_{1}$, are rows from $I_{n}$, define

$$
T_{a}=\left[\begin{array}{cc}
T_{s} & 0 \\
0 & I_{q}
\end{array}\right]
$$

where the permutation matrix $T_{s} \in \mathbb{R}^{n \times n}$ has the property that $C_{1} T_{s}^{-1}=\left[\begin{array}{cc}0 & I_{n-q}\end{array}\right]$. Applying this to the augmented system with outputs $y:=\left[\begin{array}{ll}y_{p, 1}^{T} & z_{f}^{T}\end{array}\right]^{T}$ yields a system

$$
\begin{aligned}
\dot{x}_{a}(t) & =A(\rho) x_{a}(t)+B(\rho) u_{p}(t)+D f(t)+M(\rho) \xi(\cdot) \\
y(t) & =C x_{a}
\end{aligned}
$$

where $x_{a} \in \mathbb{R}^{n+q}$ and, in particular, $C=\left[\begin{array}{ll}0 & I_{n}\end{array}\right]$ and $D=\left[\begin{array}{ll}0 & A_{f}\end{array}\right]^{T}$. Notice that, (5) now constitutes a classical unknown input formulation. Introducing (3) is a convenient way to pose the problem so that SMOs can be deployed [14]. The selection of a good choice of $A_{f}$ is important as it impacts on the performance of the system with respect to any unmatched uncertainties [14]. The system in (5) will be used as the starting point for the design of an observer.

The sliding mode observer considered here has the structure

$$
\dot{z}(t)=A(\rho) z(t)+B(\rho) u_{p}(t)+G_{l}(\rho) e_{y}(t)+G_{n} \nu(t)
$$

where $z \in \mathbb{R}^{q+n}$ represents the state estimate of $x_{a}$ [19]. In (6) $e_{y}(t)=C\left(z(t)-x_{a}(t)\right)$ is the output estimation error and 
$\nu(t)$ represents the discontinuous output error injection vector used to induce the sliding motion. Here as in [19],

$$
\nu=-k(t) \frac{e_{y}}{\left\|e_{y}\right\|} \quad \text { if } \quad e_{y} \neq 0
$$

where the modulation function $k(\cdot)$ will be defined later. In (5), decompose the augmented system and disturbance matrices as

$$
A(\rho):=\left[\begin{array}{ll}
A_{11}(\rho) & A_{12}(\rho) \\
A_{21}(\rho) & A_{22}(\rho)
\end{array}\right] \quad M(\rho):=\left[\begin{array}{l}
M_{1}(\rho) \\
M_{2}(\rho)
\end{array}\right]
$$

where $A_{11}(\rho) \in \mathbb{R}^{q \times q}, M_{2}(\rho) \in \mathbb{R}^{n \times k}$ and note that $A_{21}(\rho)$ has the following structure as explained in [19]:

$$
A_{21}(\rho)=\left[\begin{array}{c}
A_{211}(\rho) \\
A_{212}
\end{array}\right]
$$

where $A_{211}(\rho) \in \mathbb{R}^{(n-q) \times q}$ and $A_{212} \in \mathbb{R}^{q \times q}$. Define the observer gains in (6) as

$$
G_{l}(\rho)=\left[\begin{array}{c}
A_{11}(\rho) L-A_{12}(\rho)+k_{2} L \\
-A_{22}(\rho)+A_{21}(\rho) L-k_{2} I
\end{array}\right], \quad G_{n}=\left[\begin{array}{c}
-L \\
I_{n}
\end{array}\right]
$$

where $k_{2}$ is a positive design scalar and the design freedom $L \in \mathbb{R}^{q \times n}$ has the structure

$$
L=\left[\begin{array}{ll}
L_{1} & 0
\end{array}\right]
$$

and $L_{1} \in \mathbb{R}^{q \times(n-q)}$.

Assumption 3.1: The pair $\left(A_{11}(\rho), A_{211}(\rho)\right)$ is assumed to be quadratically detectable, i.e. there exists a s.p.d matrix $P_{1}$ and a gain $L_{1}$ such that

$$
P_{1}\left(A_{11}(\rho)+L_{1} A_{211}(\rho)\right)+\left(A_{11}(\rho)+L_{1} A_{211}(\rho)\right)^{T} P_{1}<0
$$

for all $\rho \in \Omega$. It can be shown that $\left(A_{11}(\rho), A_{211}(\rho)\right)$ is quadratically detectable if $\left(A_{p}(\rho), C_{1}\right)$ is quadratically detectable.

Define the state estimation error as $e=z-x_{a}=\left[\begin{array}{ll}e_{1}^{T} & e_{y}^{T}\end{array}\right]^{T}$, where $e_{1} \in \mathbb{R}^{q}$, then define another coordinate transformation $\tilde{e}=T_{L} e$ where

$$
T_{L}=\left[\begin{array}{cc}
I_{q} & L \\
0 & I_{n}
\end{array}\right]
$$

and as a result $\tilde{e}=\left[\begin{array}{ll}\tilde{e}_{1}^{T} & e_{y}^{T}\end{array}\right]^{T}$ where $\tilde{e}_{1}=e_{1}+L e_{y}$. From Assumption 3.1, as argued in [19], for any positive $k_{2}$, there exists a positive scalar $p_{2}>0$ such that $P=\operatorname{diag}\left(P_{1}, p_{2} I_{n}\right)$ satisfies

$$
P \tilde{A}_{e}(\rho)+\tilde{A}_{e}(\rho)^{T} P+Q_{0}<0 \quad \forall \rho \in \Omega
$$

where $Q_{0}>0$ is s.p.d and $\tilde{A}_{e}(\rho)=T_{L}\left(A(\rho)+G_{l}(\rho) C\right) T_{L}^{-1}$. Choose $\eta_{1}(\cdot)$, depending on the upper bound on the faults as

$$
\|\xi\| \leq c_{1}\left\|x_{p}\right\|+d \leq \eta_{1}\left(y_{p}, d\right)
$$

Let $a_{21}(t)=\left\|\tilde{A}_{21}(\rho)\right\|, m_{2}(t)=\left\|M_{2}(\rho)\right\|$ and define the scalar function $\chi(t)$ to be the solution of

$$
\dot{\chi}(t)=-q_{0} \chi(t)+\left\|P^{\frac{1}{2}} T_{L} M(\rho)\right\| \eta_{1}\left(y_{p}, d\right), \quad \chi(0)=0
$$

where the scalar $q_{0}=\frac{1}{2} \lambda_{\min }\left(P^{-\frac{1}{2}} Q_{0} P^{-\frac{1}{2}}\right)>0$ and $\lambda_{\min }(\cdot)$ denotes the minimum eigenvalue. Finally define

$$
\tilde{\chi}(t):=\left(\chi(t)+\chi_{0}\right) / \sqrt{\lambda_{\min }(P)}
$$

where $\chi_{0}$ is a positive design scalar. As argued in [19], $\tilde{\chi}(t)$ in (17) is an upper bound on the evaluation of the state estimation error $\|\tilde{e}(t)\|$, i.e. there exists a $t_{0} \geq 0$ such that for all $t \geq t_{0}$

$$
\tilde{\chi}(t) \geq\|\tilde{e}(t)\| \geq\left\|\tilde{e}_{1}(t)\right\|
$$

Since $\tilde{\chi}(t)$ is known, define the modulation function in (7) as

$$
k(t)=a_{21}(t) \tilde{\chi}(t)+\left\|A_{f}\right\| \alpha+m_{2}(t) \eta_{1}\left(y_{p}, d\right)+\eta
$$

where $\eta$ is a positive scalar. From [19], a sliding motion on

$$
\mathcal{S}_{o}=\left\{\tilde{e}(t) \in \mathbb{R}^{n+q}: C \tilde{e}(t)=e_{y}(t)=0\right\}
$$

can be enforced in finite time. Consider as a fault estimate

$$
\hat{f}=W \nu_{e q}
$$

where $\nu_{e q}$ is the equivalent output error injection signal [19] and the gain $W=\left[\begin{array}{ll}0 & A_{f}^{-1}\end{array}\right]$ where $A_{f}$ is the system matrix of the filter in (3). It can be shown during sliding [19] that

$$
\begin{aligned}
& \dot{\tilde{e}}_{1}=\tilde{A}_{11}(\rho) \tilde{e}_{1}-\tilde{M}_{1}(\rho) \xi \\
& e_{f}=-A_{f}^{-1} A_{212} \tilde{e}_{1}
\end{aligned}
$$

where the fault estimation error $e_{f}=\hat{f}-f$, the system matrix $\tilde{A}_{11}(\rho)=A_{11}(\rho)+L_{1} A_{211}(\rho)$ and $\tilde{M}_{1}(\rho)=M_{1}(\rho)+$ $L M_{2}(\rho)$. When there is no uncertainty $\xi=0$ and $\tilde{e}_{1} \rightarrow 0$ asymptotically. Consequently $e_{f} \rightarrow 0$ as $t \rightarrow \infty$. However in the presence of uncertainty, the fault estimation error no longer tends to zero.

\section{Controller Design And Closed-Loop AnAlysis}

To introduce tracking in the controller in Fig. 1, integrator states will be introduced associated with the controlled outputs

$$
y_{c}(t)=C_{c} x_{p}(t)
$$

where $C_{c} \in \mathbb{R}^{l \times n}$. In the FTC scheme as shown in Fig. 1, the estimated sensor fault is used to correct the measured states and this 'corrected' version of (23) is defined as

$$
\hat{x}_{p}(t)=x_{p}(t)+H_{p} f(t)-H_{p} \hat{f}(t)=x_{p}(t)-H_{p} e_{f}(t)
$$

Consequently the integral action states evolve according to

$$
\dot{x}_{r}(t)=r(t)-C_{c} \hat{x}_{p}(t)=r(t)-C_{c} x_{p}(t)+C_{c} H_{p} e_{f}(t)
$$

where $r(t)$ is the (differentiable) command signal which satisfies the pre-filter equations

$$
\dot{r}(t)=\Gamma\left(r(t)-R_{c}\right)
$$

In (26), $R_{c}$ represents a fixed exogenous command reference vector and $\Gamma \in \mathbb{R}^{l \times l}$ is a stable design matrix. Let $x_{c}(t)=\left[\begin{array}{ll}x_{r}^{T}(t) & x_{p}^{T}(t)\end{array}\right]^{T}$ and consider the augmented state space system obtained from combining (1) and (25):

$\dot{x}_{c}(t)=A_{c}(\rho) x_{c}(t)+B_{c}(\rho) u_{p}(t)+B_{r} r(t)+M_{c}(\rho) \xi(\cdot)+H_{c} e_{f}(t)$

where $A_{c}(\rho), B_{c}(\rho), B_{r}, M_{c}(\rho)$ are of appropriate dimensions and the gain

$$
H_{c}=\left[\begin{array}{ll}
\left(C_{c} H_{p}\right)^{T} & 0
\end{array}\right]^{T}
$$


The definition of these matrices can be found in [5]. Here as in [5], it is assumed that $B_{c}(\rho)$ can be factorized as

$$
B_{c}(\rho)=B_{v} B_{2}(\rho)
$$

where $B_{v} \in \mathbb{R}^{(l+n) \times l}$ is a fixed matrix, and $B_{2}(\rho) \in \mathbb{R}^{l \times m}$ is a matrix with varying components where $\operatorname{rank}\left(B_{2}(\rho)\right)=l$ for all $\rho \in \Omega$ and $\operatorname{rank}\left(B_{v}\right)=l$. Since $\operatorname{rank}\left(B_{v}\right)=l$ there exists a coordinate change $x_{c} \mapsto T_{c} x_{c}=\tilde{x}$ where

$$
T_{c}=\left[\begin{array}{cc}
I_{l} & 0 \\
0 & T_{n}
\end{array}\right]
$$

and $T_{n} \in \mathbb{R}^{n \times n}$ is a nonsingular matrix with the property that

$$
\tilde{B}_{v}=T_{c} B_{v}=\left[\begin{array}{l}
0 \\
I_{l}
\end{array}\right]
$$

In the new coordinate system, (27) can be written as

$$
\dot{\tilde{x}}(t)=\tilde{A}_{c}(\rho) \tilde{x}(t)+\tilde{B}_{v} \tilde{v}(t)+\tilde{B}_{r} r(t)+\tilde{M}_{c}(\rho) \xi(\cdot)+\tilde{H}_{c} e_{f}(t)
$$

where $\tilde{H}_{c}=H_{c}$ and the virtual control $\tilde{v}(t) \in \mathbb{R}^{l}$ satisfies

$$
\tilde{v}(t)=B_{2}(\rho) u_{p}(t)
$$

To analyse the closed-loop under FTC, define

$$
\hat{x}(t)=\tilde{x}(t)+\underbrace{\left[\begin{array}{c}
0 \\
T_{n} H_{p}
\end{array}\right]}_{\tilde{H}_{p}} e_{f}(t)
$$

where $T_{n}$ is defined in (30). Then from (32) and (34)

$$
\begin{aligned}
\dot{\hat{x}}(t)=\tilde{A}_{c}(\rho) \hat{x}(t) & +\tilde{B}_{v} \tilde{v}(t)+\tilde{B}_{r} r(t)+\tilde{M}_{c}(\rho) \xi(\cdot) \\
& +\left(\tilde{H}_{c}-\tilde{A}_{c}(\rho) \tilde{H}_{p}\right) e_{f}(t)+\tilde{H}_{p} \dot{e}_{f}
\end{aligned}
$$

Since from (22)

$$
\dot{e}_{f}=-A_{f}^{-1} A_{212} \dot{\tilde{e}}_{1}=-A_{f}^{-1} A_{212}\left(\tilde{A}_{11}(\rho) \tilde{e}_{1}-\tilde{M}_{1}(\rho) \xi\right)
$$

it follows that equation (35) can be written as

$$
\dot{\hat{x}}=\tilde{A}_{c}(\rho) \hat{x}+\tilde{B}_{v} \tilde{v}+\tilde{B}_{r} r+\hat{M}(\rho) \xi(\cdot)+\hat{H}(\rho) \tilde{e}_{1}
$$

where

$$
\begin{aligned}
& \hat{H}(\rho)=\left(\tilde{A}_{c}(\rho) \tilde{H}_{p}-\tilde{H}_{c}\right) A_{f}^{-1} A_{212}-\tilde{H}_{p} A_{f}^{-1} A_{212} \tilde{A}_{11}(\rho) \\
& \hat{M}(\rho)=\tilde{M}_{c}(\rho)+\tilde{H}_{p} A_{f}^{-1} A_{212}\left(M_{1}(\rho)+L M_{2}(\rho)\right)
\end{aligned}
$$

Here a parameter-dependent switching function is defined as

$$
\hat{s}(t)=S(\rho) \hat{x}(t)
$$

where $S(\rho)=\left[N(\rho) \quad I_{l}\right]$ and $N(\rho)$ represents the design freedom. Clearly $S(\rho) B_{v}=I_{l}$ and it follows from (37) that

$$
\begin{aligned}
\dot{\hat{s}}(t)= & S(\rho)\left(\tilde{A}_{c}(\rho) \hat{x}(t)+\tilde{B}_{r} r(t)+\hat{M}(\rho) \xi(\cdot)+\hat{H}(\rho) \tilde{e}_{1}(t)\right) \\
& +\tilde{v}(t)+\dot{S}(\rho) \hat{x}(t)
\end{aligned}
$$

Choose the sliding mode control law as in [5]

$$
\tilde{v}(t)=\tilde{v}_{l}(t)+\tilde{v}_{n}(t)
$$

where

$$
\tilde{v}_{l}(t)=-S(\rho)\left(\tilde{A}_{c}(\rho) \hat{x}(t)+\tilde{B}_{r} r(t)\right)-\dot{S}(\rho) \hat{x}(t)+\Phi \hat{s}(t)
$$

and $\Phi \in \mathbb{R}^{l \times l}$ is a Hurwitz matrix. The component $\tilde{v}_{n}(t)$ will be defined in the sequel. It follows from (40) and (42) that

$$
\dot{\hat{s}}(t)=\tilde{v}_{n}(t)+\Phi \hat{s}(t)+S(\rho)\left(\hat{M}(\rho) \xi(\cdot)+\hat{H}(\rho) \tilde{e}_{1}(t)\right)
$$

Note that in (43) whilst $\xi$ and $\tilde{e}_{1}$ are unknown, upper bounds on $\|\xi\|$ and $\left\|\tilde{e}_{1}\right\|$, used to construct the modulation function $k(t)$ in (19), are known and defined in (15) and (18). Let

$$
\tilde{v}_{n}(t)=-\mathcal{K}\left(t, y_{p}\right) \frac{P_{2} \hat{s}(t)}{\left\|P_{2} \hat{s}(t)\right\|} \quad \text { if } \quad \hat{s}(t) \neq 0
$$

where the symmetric positive definite matrix $P_{2}$ satisfies

$$
P_{2} \Phi+\Phi^{T} P_{2}=-I_{l}
$$

A suitable modulation gain $\mathcal{K}\left(t, y_{p}\right)$ is given by

$$
\mathcal{K}\left(t, y_{p}\right)=m(t) \eta_{1}\left(y_{p}, d\right)+h(t) \tilde{\chi}(t)+\eta_{2}
$$

where $m(t)=\|S(\rho) \hat{M}(\rho)\|, h(t)=\|S(\rho) \hat{H}(\rho)\|$ and $\eta_{2}$ denotes a positive scalar. Note that since $\Omega$ is compact, $\rho$ is bounded and therefore both $m(t)$ and $h(t)$ are bounded since both $\hat{M}(\rho)$ and $\hat{H}(\rho)$ are affine with respect to $\rho$.

Proposition 4.1: The choice of the modulation gain in (46) ensures sliding takes palace on

$$
\mathcal{S}_{c}=\{\hat{x}: S(\rho) \hat{x}=0\}
$$

Proof: Define a candidate Lyapunov equation according to $\hat{V}=\frac{1}{2} \hat{s}^{T} P_{2} \hat{s}$. It follows from (43) and (44) that

$$
\begin{aligned}
\dot{\hat{V}} & =\hat{s}^{T} P_{2}\left(\tilde{v}_{n}+\Phi \hat{s}+S(\rho) \hat{M}(\rho) \xi(\cdot)+S(\rho) \hat{H}(\rho) \tilde{e}_{1}\right) \\
& \leq\left\|P_{2} \hat{s}\right\|\left(-\mathcal{K}(t, \hat{x})+m(t) \eta_{1}\left(y_{p}, d\right)+h(t) \tilde{\chi}(t)\right)
\end{aligned}
$$

since by construction $\tilde{\chi}(t)$ in (17) is an upper bound of $\left\|\tilde{e}_{1}\right\|$. If $\mathcal{K}\left(t, y_{p}\right)$ is chosen as in (46), $\dot{\hat{V}} \leq-\eta_{2}\left\|P_{2} \hat{s}\right\|$ and this ensures sliding takes place in finite time and is maintained.

Recalling the full order dynamics in (37), define

$$
\tilde{A}_{c}(\rho)=\left[\begin{array}{ll}
\tilde{A}_{c 11}(\rho) & \tilde{A}_{c 12}(\rho) \\
A_{c 21}(\rho) & A_{c 22}(\rho)
\end{array}\right] \quad \hat{M}(\rho)=\left[\begin{array}{l}
\hat{M}_{1}(\rho) \\
\hat{M}_{2}(\rho)
\end{array}\right]
$$

and

$$
\hat{H}(\rho)=\left[\begin{array}{c}
\hat{H}_{1}(\rho) \\
\hat{H}_{2}(\rho)
\end{array}\right] \quad \tilde{B}_{r}=\left[\begin{array}{c}
\tilde{B}_{r 1} \\
0
\end{array}\right]
$$

In (49), $\tilde{A}_{c 11}(\rho) \in \mathbb{R}^{n \times n}$ and the other matrices are partitioned conformal with this. Also define $\hat{x}=\left[\begin{array}{ll}\hat{x}_{1}^{T} & \hat{x}_{2}^{T}\end{array}\right]^{T}$ where $\hat{x}_{2} \in \mathbb{R}^{l}$.

Assumption 4.1: Assume there exists a s.p.d matrix $\tilde{P}_{1}$ and a gain $N(\rho)$ so that for all $\rho \in \Omega$

$\tilde{P}_{1}(\underbrace{\tilde{A}_{c 11}(\rho)-\tilde{A}_{c 12}(\rho) N(\rho)}_{\hat{A}_{11}^{s}(\rho)})+\left(\tilde{A}_{c 11}(\rho)-\tilde{A}_{c 12}(\rho) N(\rho)\right)^{T} \tilde{P}_{1}<0$

Remark 4.1: The synthesis of $N(\rho)$ is based on an LMI formulation involving the vertices of $\tilde{A}_{c}(\rho)$ [17]. Here if the vertices of $A_{p}(\rho)$ are written as $\left\{A_{p}^{i}\right\}$ for $i=1, \ldots, 2^{r}$ and $B_{v p}$ represents the bottom $n$ rows of $B_{v}$, then provided each of the LTI systems $\left(A_{p}^{i}, B_{v p}, C_{c}\right)$ for $i=1, \ldots, 2^{r}$ does not have any invariant zeros at the origin, the algorithm in [17] can be employed. (The presence of invariant zeros at the origin causes pole/zero cancellation during the augmentation of the integral action states.) 
During sliding on $\mathcal{S}_{c}$, from (39), $\hat{x}_{2}=-N(\rho) \hat{x}_{1}$ and the reduced order motion is given by

$$
\dot{\hat{x}}_{1}=\hat{A}_{11}^{s}(\rho) \hat{x}_{1}+\hat{H}_{1}(\rho) \tilde{e}_{1}+\hat{M}_{1}(\rho) \xi+\tilde{B}_{r 1} r
$$

Define $\hat{z}=\left[\begin{array}{cc}\hat{x}_{1}^{T} & \tilde{e}_{1}^{T}\end{array}\right]^{T}$, then it follows the overall dynamics during sliding are governed by (22) and (51) and can be written

$$
\dot{\hat{z}}=\underbrace{\left[\begin{array}{cc}
\hat{A}_{11}^{s}(\rho) & \hat{H}_{1}(\rho) \\
0 & \tilde{A}_{11}(\rho)
\end{array}\right]}_{A_{s}(\rho)} \hat{z}+\underbrace{\left[\begin{array}{c}
\hat{M}_{1}(\rho) \\
\tilde{M}_{1}(\rho)
\end{array}\right]}_{M_{s}(\rho)} \xi+\left[\begin{array}{c}
\tilde{B}_{r 1} \\
0
\end{array}\right] r
$$

In the presence of uncertainty, since by assumption $\xi$ is cone bounded with respect to $x_{p}$, and hence to $\hat{z}$, a small gain approach will be adopted to prove stability. By construction

$$
x_{c}=T_{c}^{-1} \tilde{x}=\left[\begin{array}{cc}
I_{l} & 0 \\
0 & T_{n}^{-1}
\end{array}\right] \tilde{x}
$$

and then since $x_{p}$ represents the last $n$ rows of $x_{c}$ as shown in (27), it follows

$$
x_{p}=\left[\begin{array}{ll}
0 & T_{n}^{-1}
\end{array}\right] \tilde{x}
$$

By definition

$$
\tilde{x}=\hat{x}+\tilde{H}_{p} A_{f}^{-1} A_{212} \tilde{e}_{1}
$$

then during sliding, since $\hat{x}_{2}=-N(\rho) \hat{x}_{1}$, it follows that

$$
\hat{x}=\left[\begin{array}{c}
I \\
-N(\rho)
\end{array}\right] \hat{x}_{1}
$$

Therefore from (54)-(56)

$$
x_{p}=\underbrace{\left[\begin{array}{ll}
0 & T_{n}^{-1}
\end{array}\right]\left[\left[\begin{array}{c}
I \\
-N(\rho)
\end{array}\right] \mid \tilde{H}_{p} A_{f}^{-1} A_{212}\right]}_{C_{s}(\rho)}\left[\frac{\hat{x}_{1}}{\tilde{e}_{1}}\right]
$$

Since by assumption $\|\xi\|<c_{1}\left\|x_{p}\right\|+c_{2}(t)$, the stability of (52) result follows from the use of the small gain theorem, if

$$
\mathcal{G}:\left\{\begin{array}{l}
\dot{\hat{z}}=A_{s}(\rho) \hat{z}+M_{s}(\rho) \xi \\
x_{p}=C_{s}(\rho) \hat{z}
\end{array}\right.
$$

has an $\mathcal{L}_{2}$ gain no more than $1 / c_{1}$. Now the problem becomes easy to solve by directly applying the Bounded Real Lemma to the LPV system in (58); and $L$ and the vertices of $N(\rho)$ can be calculated by solving a finite number of LMIs [20].

\section{OBSERVER AND CONTROLlER DESIGN RESUlTS}

The theoretical developments in Sections II-IV were employed to create an SMO based sensor fault FTC scheme for JAXA's MuPAL- $\alpha$ aircraft (see Fig. 3). The MuPAL- $\alpha$ is based on the twin-propeller engine Dornier Do228-202 aircraft modified to include a research FBW system and Direct Lift Control (DLC) flaps [8], [21]. An LPV model of MuPAL- $\alpha$ was created via interpolation of LTI models verified from real flight data provided by JAXA. The scheduling parameters are chosen as

$$
\rho=\left[\begin{array}{ll}
\rho_{1} & \rho_{2}
\end{array}\right]:=\left[\begin{array}{ll}
v_{\text {eas }} & v_{\text {eas }}^{2}
\end{array}\right]
$$

where $v_{\text {eas }}$ represents the equivalent airspeed [21]. Regulatory constraints mean usually the test flights take place at a similar altitude and the aircraft set up in terms of mass and the position of the centre of gravity do not change significantly.
Therefore, the scheduling parameter is set only as $v_{\text {eas }}$. For design purposes, the conservative assumption was made that the scheduling parameters $\rho_{1}$ and $\rho_{2}$ are independent of each other. This paper focuses on the lateral-directional dynamics.

After re-ordering the system outputs so that the sensor considered potentially prone to faults (i.e. the yaw rate sensor) appears as the last component of the output vector, the system states are roll angle $\phi$, sideslip angle $\beta$, roll rate and yaw rate. In this paper, it is assumed that all system states are measured. In (1), the system inputs $u_{p}$ are given by $u_{p}=\left[\begin{array}{ll}\delta_{a} & \delta_{r}\end{array}\right]^{T}$ where $\delta_{a}$ and $\delta_{r}$ represent the aileron and rudder surface deflections, respectively. Consequently, $n=4, q=1$. The disturbance distribution matrix $M_{p}(\rho)$ is assumed to be fixed and to take the form

$$
M_{p}(\rho)=\left[\begin{array}{ll}
0_{2 \times 2} & I_{2}
\end{array}\right]^{T}
$$

which models uncertainty/disturbances $\xi(\cdot)$ affecting the roll rate and yaw rate channels. Here the matrix is used as (indirect) design freedom during the synthesis of $L$ from (11).

Remark 5.1: For this testing campaign, pragmatically, 'channels' in the system representation in (1) have been established/chosen to capture the plant-model mismatch. In such a situation, parameterizing the uncertainty channels in terms of $\rho$ provides no great added value. Consequently, here, the fixed matrix $M$ represents the 'channels', and the effect of changes in $\rho$ are incorporated into the uncertainty signal $\xi$. In terms of the observer design, the filter parameter $A_{f}=$ 0.01 . The bandwidth of the filter is important and affects the robustness of the fault estimate: for a detailed discussion see page 216 in [14]. The scalar $k_{2}$ in (10) is chosen as $k_{2}=0.1$, and $L$ from (11) is calculated using YALMIP with the SeDuMi LMI solver. For this particular design an upper-bound on the $\mathcal{L}_{2}$ gain in (58), computed via the Bounded Real Lemma, is 1.2918. Here because the design freedom $N(\rho)$ is chosen as fixed, the representation in (58) is affine with respect to $\rho$. An LMI approach ([20], [5]) has been used to compute the upperbound on the $\mathcal{L}_{2}$ gain. This gain gives a robustness margin to the uncertainty structure in which $c_{1}=1 / 1.2918$.

The observer modulation gain $k(t)$ in (7) was chosen as 0.8. The small positive scalar selected for the sigmoidal approximation [17] to the discontinuous injection term in the observer in (7) is 0.01 . The modulation gain must be chosen large enough to ensure a sliding motion can be maintained even in the presence of faults. This requires a-priori knowledge of the level of faults the scheme will be able to handle and provide fault tolerance. An unnecessarily large value of $k(t)$ will increase the level of chattering and will amplify noise and will make the extraction of a smooth equivalent output error injection signal more difficult to obtain. Typically these values would be tuned during simulations. The modulation gain $k(t)$ comprises three main elements: a term to bound the evolution of the unknown error $e_{1}(t)$ (particularly initially mismatches between the initial conditions of the observer and their unknown true plant values), a bound on the lumped disturbances capturing the plant model mismatches and any unmodelled external disturbance $\xi$ impacting on the plant; and a term to bound the magnitude of the unknown faults. 
In this example the error term $e_{1}(t)$ is a scalar and has physical meaning, and so in this situation it is possible to make a good initial guess for the initialization of the observer to ensure $e_{1}(0)$ is small. Thus the dominating terms are associated with the evaluation of $e_{1}(t)$ resulting from the plant model mismatched captured by $\xi$ and the size of the faults. To estimate the effects of plant model mismatch, the observer was run (off-line) on flight data obtained from the experiments in [5] which constitutes real sensor fault-free data. Results for different choices of $k(t)$ are illustrated in Fig. 2. This suggests a choice of $k(t)=1.2$. The remaining design freedom is associated with the allowable magnitude of sensor faults to be accommodated. Here a-priori, for safety reasons, it was determined that the magnitude of the sensor faults would be less that $5 \mathrm{deg} / \mathrm{s}$. This was used as the basis for the choice of $\alpha=0.1 \mathrm{rad}$. The final value of $k(t)$ was decided upon following extensive pre-flight ground testing on the MuPAL$\alpha$ aircraft configured in a Hardware-in-the-Loop (HIL) setup [8]. The results encapsulated in Fig. 2 represent 'open-loop' tests since the reconstructions from the observer do not affect the control signals applied to the aircraft. ('old' flight test data based on other flight experiments was used). When testing the complete FTC strategy shown in Fig. 1 on the HIL set up, the closed-loop response was found to improve if a reduced value of $k(t)$ was employed. This interaction between the FDI scheme (estimating the sensor faults) and the feedback control loop is not unexpected and arises from the lack of a true separation principle, because of the coupling introduced by the uncertainty. This coupling is a studied phenomenon in the FDI/FTC literature [27]. Ultimately a value of $k(t)=0.8$ was selected - which although not optimal with respect to Fig. 2, only represents a $12.5 \%$ degradation in performance from the open-loop optimal value near $k(t)=1.2$ but yields better closed loop performance.
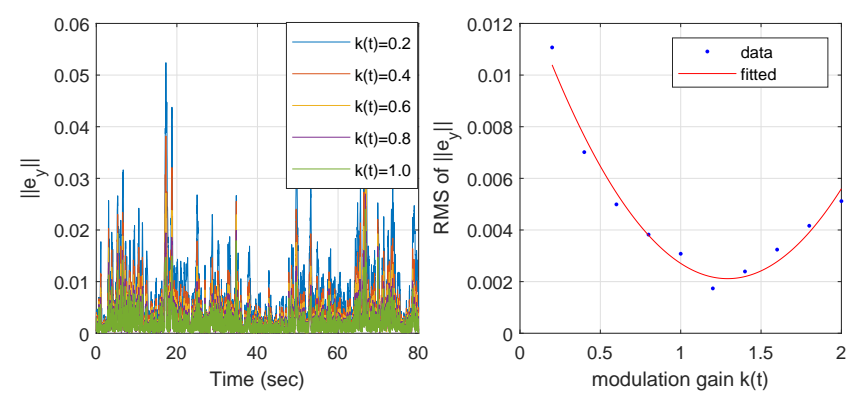

Fig. 2. An example of a fine-tuning of the modulation gain

The controller design implemented here is the one described in detail in [5]. The controller in [5] was developed specifically to provide actuator fault tolerance, but in this paper has been embedded with the scheme in Fig. 1. Extensive tuning of the controller was undertaken in the HIL environment discussed in [5]. For details of the controller tuning on the HIL and the trade-offs involved, see [5].

\section{Flight TEST ON MUPAL- $\alpha$}

The FBW configuration of MuPAL- $\alpha$ allows new flight control strategies to be implemented (on the FBW flight control computer) and then evaluated either on the ground (as part of a HIL configuration) or by actual piloted flight evaluation.

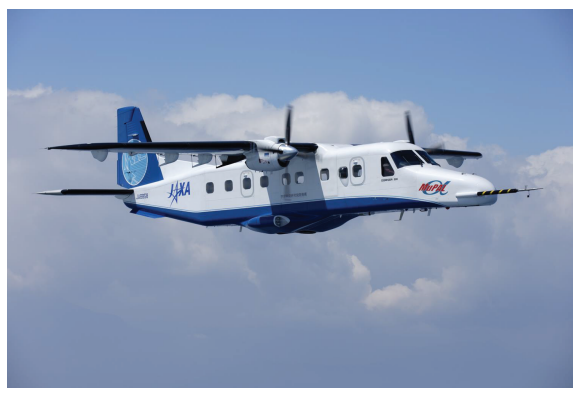

Fig. 3. MuPAL- $\alpha$ aircraft

The sensor FTC scheme (as described in Sections II-IV) was written in $\mathrm{C}$-code using a template provided by JAXA to comply with the input-output interface of the FBW system. Once HIL testing is successfully completed, the control scheme is 'burned' into the FBW computer. The sensor FTC scheme was implemented on the FBW system using an explicit Euler solver based on a sample rate of $50 \mathrm{~Hz}$.

The results presented here were obtained from flight campaigns supervised by a crew of JAXA and Nikanihon Air Service personnel, between 16-26 April 2018. The flight tests took place in Sagami Bay, south-west of Tokyo.

During the flight test campaign, the FTC sliding mode scheme developed in Sections II-IV was used to achieve lateral-directional performance. Longitudinal control of altitude and speed was manually maintained by the evaluation pilot using column and throttle lever inputs. In order to demonstrate nominal fault free performance can be retained in the presence of sensor faults, a coordinated 'S-turn' manoeuvre, involving a roll angle command of approximately $20 \mathrm{deg}$ to $-20 \mathrm{deg}$ followed by a sideslip command of $-2 \mathrm{deg}$ to $3 \mathrm{deg}$, was conducted manually by the evaluation pilot. During the flight tests, faults were set to occur $30 \mathrm{sec}$ after the FBW system was engaged.

\section{A. Sensor fault tolerant control flight tests}

1) Fault free: The flight test results are shown in Fig. 4 in the presence of wind/gusts. A coordinated 'S-turn' manoeuvre described previously was considered here. The trajectories of the aircraft states associated with the lateral-directional motion and wind/gusts are shown in Fig. 4(a). It is clear from Fig. 4(a) that sideslip and roll angle tracking performance are good. The aileron and rudder surface deflections are shown in Fig. 4(b). The observer performance is shown in Fig. 4(c) where $\left\|e_{y}\right\|$ is seen to be small which indicates good sliding mode performance, and that the observer output estimation error is small. Fig. 4(c) (bottom left) also shows the fault reconstruction error is close to zero in the fault free condition, which indicates good reconstruction performance.

2) Yaw rate sensor fault - slow drift: Here a set of results are shown when a slow drift fault occurs on the yaw rate sensor from $30 \mathrm{sec}$ onwards with a drift rate of $0.1 \mathrm{deg} / \mathrm{s}^{2}$ as shown 

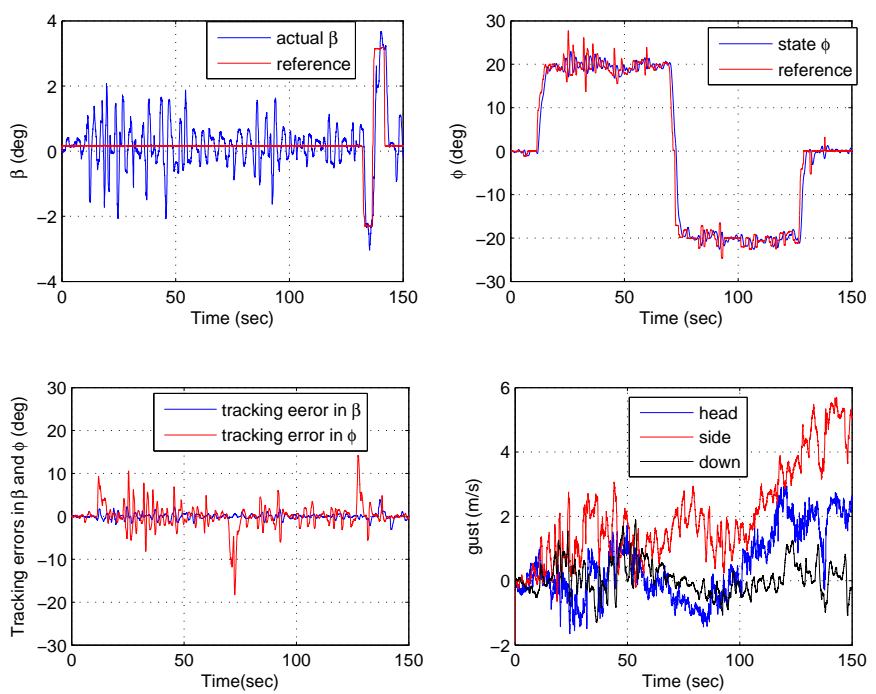

(a) The trajectories of the system states and wind/gusts
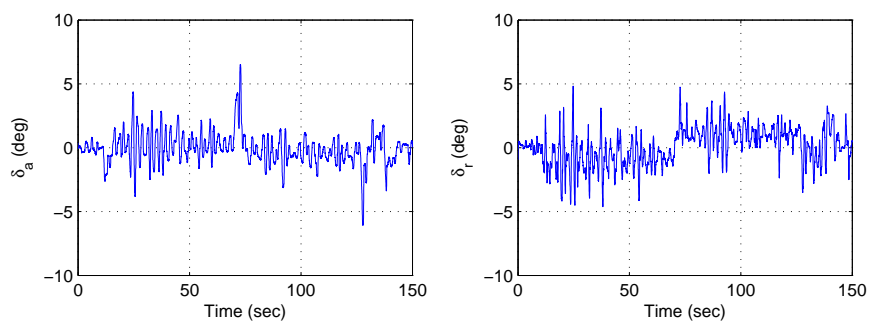

(b) Control surface deflections
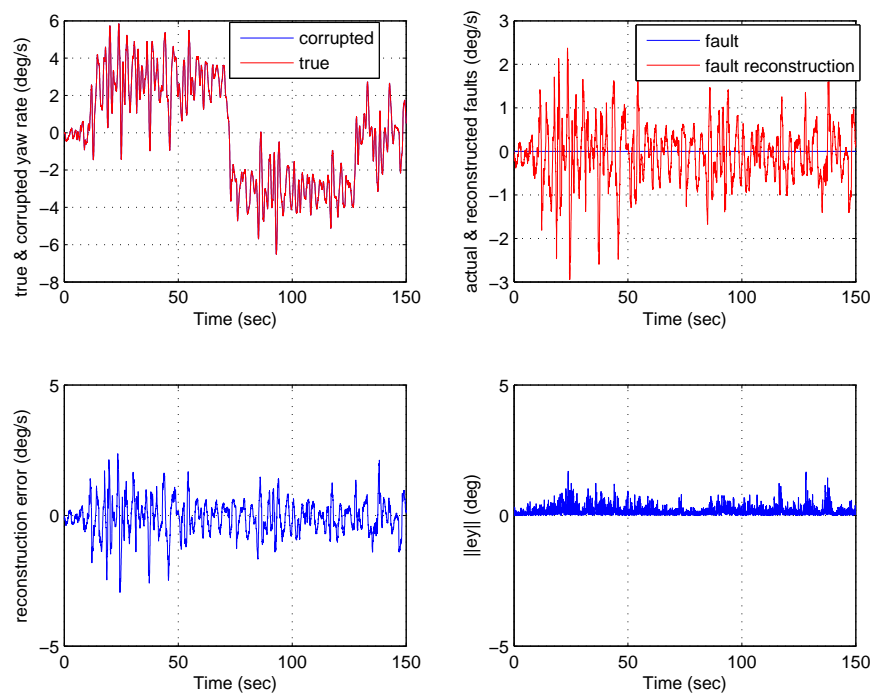

(c) Observer performance

Fig. 4. Fault-free case

in Fig. 5. For safety reasons, during flight tests, the maximum allowable amplitude of the fault is set to be $5 \mathrm{deg} / \mathrm{s}$. The trajectories of the lateral-directional states and wind/gusts are shown in Fig. 5(a) where the same coordinated 'S-turn' manoeuvres with \pm 20 deg roll angle and \pm 2 deg sideslip commands were created by the evaluation pilot. The aileron and rudder surface deflections are shown in Fig. 5(b), which are similar to the fault free case. Clearly, although a yaw rate sensor fault is present, the proposed sensor FTC scheme can still retain good roll and sideslip tracking performance (similar in level to the fault free condition). In Fig. 5(c) (top left), the 'true' yaw rate represents the actual true yaw rate experienced under the fault tolerant control scheme and the 'corrupted' signal is the sum of true yaw rate and the additive yaw rate fault. It is clear that the yaw rate sensor measurement is corrupted by a slow drift and that the fault can be well reconstructed with the fault reconstruction error close to zero (Fig. 5(c) bottom left). Furthermore, Fig. 5(c) (bottom right) shows that, during the flight tests, sliding is maintained as $\left\|e_{y}\right\|$ is close to zero.

3) Yaw rate sensor fault - fast drift: Fig. 6 shows the results associated with a fast runaway sensor fault. Similar to the slow drift in the previous test, the fault is set to occur from 30sec onwards - except now at a faster rate of $0.4 \mathrm{deg} / \mathrm{s}^{2}$. Again, for safety reasons during the flight test, the maximum amplitude of the runaway is limited to $5 \mathrm{deg} / \mathrm{s}$. The same manual pilot input coordinate ' $\mathrm{S}$-turn' is considered here for consistency. Fig. 6(a) shows the sensor fault FTC scheme managed to maintained fault-free performance in the presence of wind/gust and sensor faults. Fig. 6(a) shows good controller tracking performance, and the state responses are similar to the fault free case. The control surface deflections in Fig. 6(b) also show similar behaviour to the fault free case. Fig. 6(c) (top right) shows the fault estimation performance and demonstrates the fault reconstruction error is close to zero (bottom left) . Fig. 6(c) (bottom right) also shows that sliding motion $\left\|e_{y}\right\|$ is maintained is close to zero despite the presence of faults and wind.

4) Yaw rate sensor fault - sine wave: Fig. 7 shows the flight evaluation results when an additive sine wave fault occurs on the yaw rate sensor from $30 \mathrm{sec}$ onwards. The faulty sine wave signal has a frequency of $0.02 \mathrm{~Hz}$ and the amplitude of the fault is $2 \mathrm{deg}$. The same, manual pilot coordinated ' $\mathrm{S}$ turn' manoeuvre, is also considered here. The trajectories of the lateral-directional states of the MuPAL- $\alpha$ and associated wind/gusts are shown in Fig. 7(a) and demonstrate that fault free tracking performance of roll angle and sideslip angle can be maintained despite the yaw rate sensor corruptions. The aileron and rudder surface deflections are shown in Fig. 7(b) and indicate similar behaviour to the fault free case. Fig. 7(c) (top left) shows that the additive sine wave fault appears in the yaw rate sensor measurement from $30 \mathrm{sec}$ onwards. Nevertheless, the fault can be well reconstructed (Fig. 7(c) top right) and both the sliding surface and the fault reconstruction error are close to zero (Fig. 7(c) bottom left and right).

5) Pilot feedback: After debriefing, the evaluation pilot indicated that he did not perceive any noticeable difference in performance during the flight tests, regardless of whether the tests were fault free or included fault conditions. Note that the exact time when sensor fault occurred was not disclosed to the pilot to avoid the pilot anticipating the fault and affecting the judgement of the pilot.

\section{CONCLUSION}

This paper has developed a new LPV sliding mode sensor FTC scheme which aims to maintain near nominal lateral- 

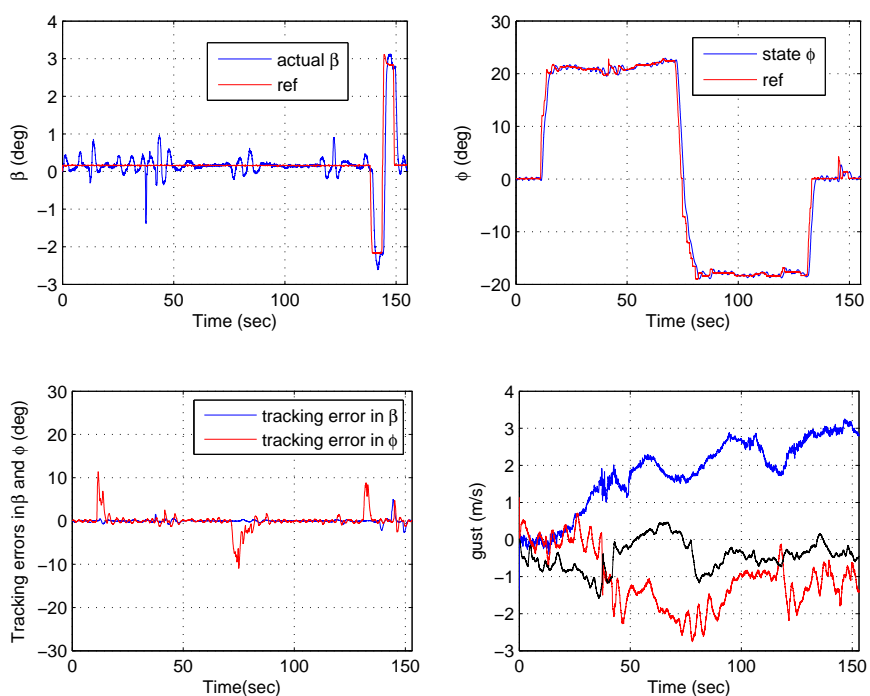

(a) The trajectories of the system states and wind/gusts
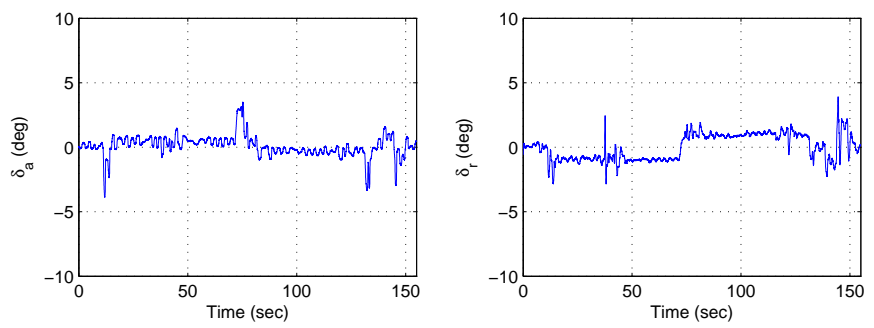

(b) Control surface deflections
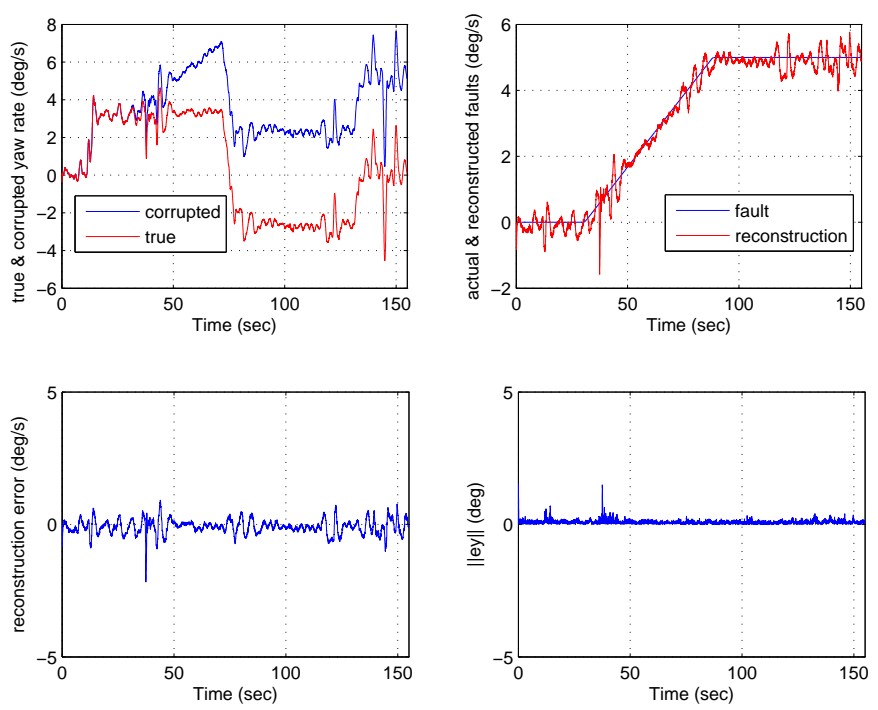

(c) Observer performance

Fig. 5. Slow drift fault

directional control performance in the face of yaw rate sensor faults. This is achieved based on only knowledge from measured system inputs and outputs. The proposed scheme has been implemented on the Japan Aerospace Exploration Agency's Multi-Purpose Aviation Laboratory (MuPAL- $\alpha$ ) research aircraft and validated in a series of flight tests. The illustrated flight test results show that, during the steady turn
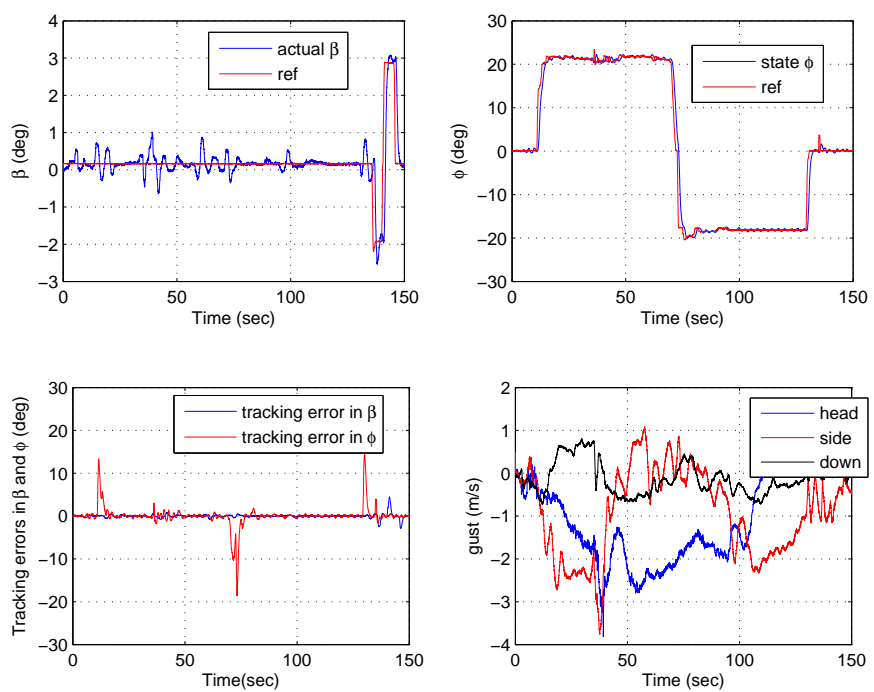

(a) The trajectories of the system states and wind/gusts
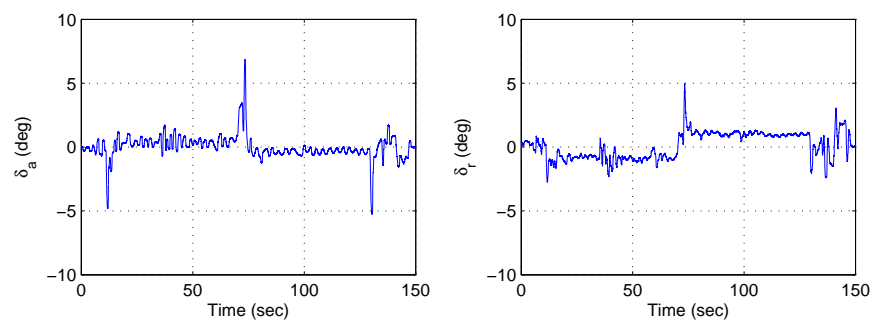

(b) Control surface deflections
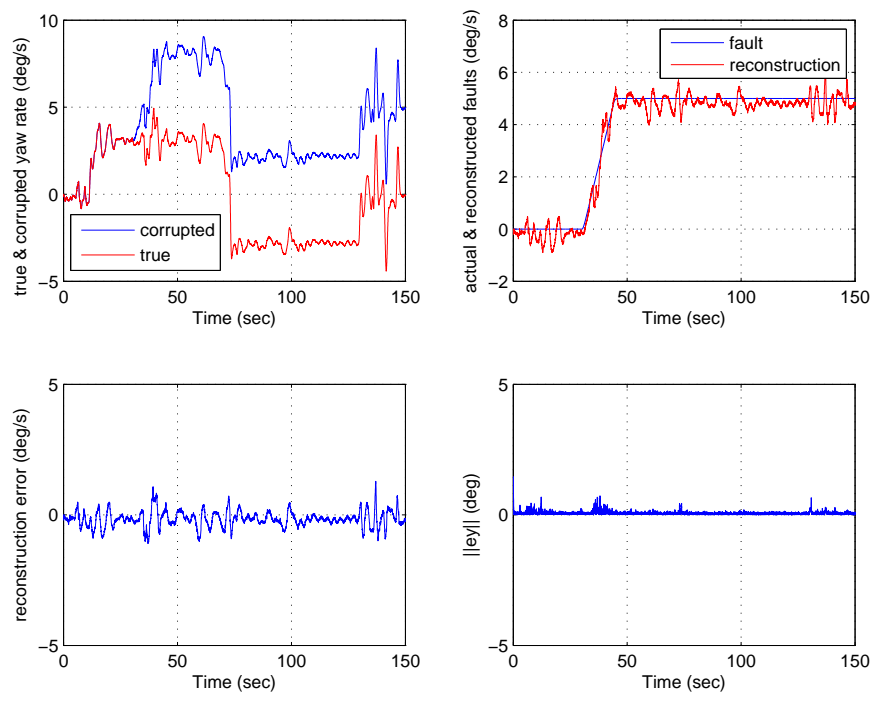

(c) Observer performance

Fig. 6. Fast drift fault

manoeuvre induced by the evaluation pilot, various yaw rate sensor faults are accurately reconstructed and roll and sideslip tracking performance is maintained despite the faults.

\section{ACKNOWLEDGEMENTS}

This work is funded by the European Union Horizon 2020 research and innovation programme under grant agreement 

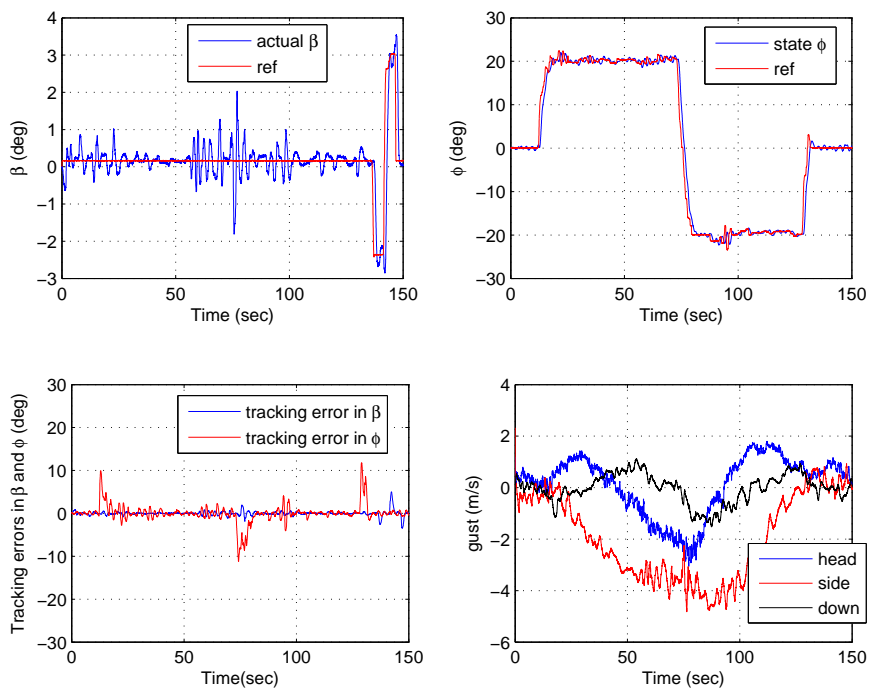

(a) The trajectories of the system states and wind/gusts
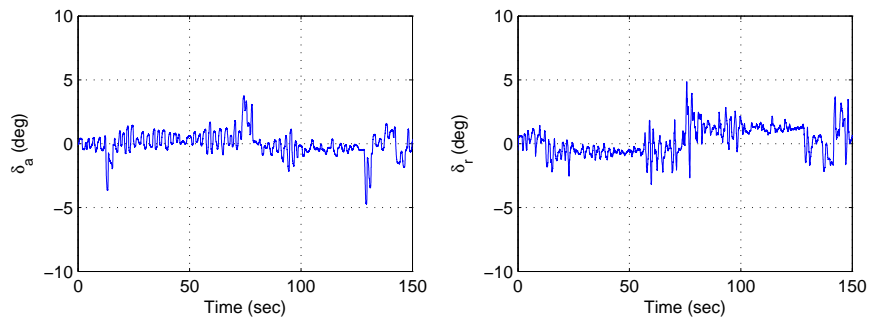

(b) Control surface deflections
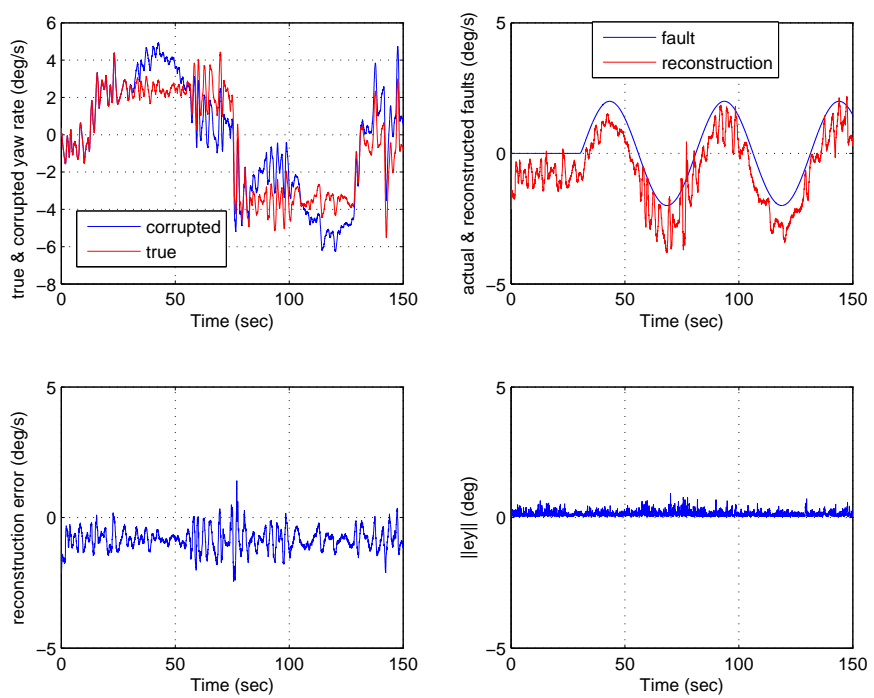

(c) Observer performance

Fig. 7. Sine wave fault

No. 690811 and the Japan New Energy and Industrial Technology Development Organization under grant agreement No. 062800, as part of the EU/Japan joint research project entitled 'Validation of Integrated Safety-enhanced Intelligent flight cONtrol (VISION)'.

\section{REFERENCES}

[1] M. Blanke, M. Kinnaert, J. Lunze, and M. Staroswiecki, Introduction to Diagnosis and Fault-Tolerant Control. Springer Berlin Heidelberg, 2016.

[2] C. Edwards, T. Lombaerts, and H. Smaili, Fault tolerant fight control: A benchmark challenge. Springer, 2010.

[3] P. Goupil and A. Marcos, "The European ADDSAFE project: Industrial and academic efforts towards advanced fault diagnosis," Control Engineering Practice, 31, pp. 109-125, 2014.

[4] P. Goupil, J. Boada-Bauxell, A. Marcos, P. Rosa, M. Kerr, and L. Dalbies, "An overview of the FP7 RECONFIGURE project: Industrial, scientific and technological objectives," in SAFEPROCESS '15, 2015.

[5] L. Chen, H. Alwi, C. Edwards and M. Sato, "Flight evaluation of a sliding mode online control allocation scheme for fault tolerant control," Automatica, 114, 2020.

[6] D. Henry, J. Cieslak, A. Zolghadri, and D. Efimov, "A non-conservative solution for early and robust fault diagnosis in aircraft control surface servo-loops," Control Engineering Practice, 31, pp. 183-199, 2014.

[7] L. Van Eykeren and Q. P. Chu, "Sensor fault detection and isolation for aircraft control systems by kinematic relations," Control Engineering Practice, 31, pp. 200-210, 2014.

[8] K. Masui and Y. Tsukano, "Development of a new in-flight simulator MuPAL- $\alpha, "$ AIAA paper 2000-4574, Aug. 2000.

[9] G. Hardier and G. Ferreres and M. Sato, "Design and Flight Testing of an Adaptive Gain-Scheduled Controller Using On-Line Model Estimation," IEEE CCTA, pp. 766-773, 2018.

[10] S. Waitman, A. Marcos and M. Sato, "Design and Hardware-In-the-Loop Validation of a Fault-Tolerant Y* Flight Control Law," Systol, 2019.

[11] H. Alwi and C. Edwards, "Development and application of sliding mode LPV fault reconstruction schemes for the ADDSAFE Benchmark," Control Engineering Practice, 31, pp. 148-170, 2014.

[12] B. Vanek, A. Edelmayer, Z. Szabo, and J. Bokor, "Bridging the gap between theory and practice in LPV fault detection for flight control actuators," Control Engineering Practice, 31, pp. 171-182, 2014.

[13] H. Alwi and C. Edwards, "Fault detection and fault-tolerant control of a civil aircraft using a sliding-mode-based scheme," IEEE Trans. Control Syst. Technol., 16, pp.499-510, 2008.

[14] H. Alwi, C. Edwards, and C. P. Tan, Fault Detection and Fault-Tolerant Control Using Sliding Modes. Springer, 2011.

[15] H. Khalil and L. Praly, "High-gain observers in nonlinear feedback control," Int. J. Robust Nonlinear Control, 24, pp.993-1015, 2010.

[16] J. Stoustrup and H. H. Niemann, "Fault estimation - a standard problem approach," Int. J. Robust Nonlinear Control, 12, pp.649-673, 2002.

[17] C. Edwards and S. K. Spurgeon, Sliding Mode Control: Theory and Applications. Taylor \& Francis, 1998.

[18] P. Goupil, R. Dayre, P. Brot, "From theory to flight tests: Airbus Flight Control System TRL5 achievements," IFAC World Congress, 2014.

[19] L. Chen, H. Alwi and C. Edwards,"On the Synthesis of an Integrated Active LPV FTC Scheme using Sliding Modes," Automatica, 110, 2019.

[20] D. Rotondo, F. Nejjari, V. Puig, and J. Blesa, "Model reference FTC for LPV systems using virtual actuator and set-membership fault estimation," Int. J. Robust Nonlinear Control, 25, pp.753-60, 2015.

[21] M. Sato, "Robust Gain-Scheduled Flight Controller for an In-Flight Simulator," IEEE Trans. Aerosp. Electron. Syst.,56, pp.2122-2135, 2020.

[22] L. Chen, C. Edwards and H. Alwi, "Sensor fault estimation using LPV sliding mode observers with erroneous scheduling parameters", Automatica, 101, 2019.

[23] A. Ferreira De Loza, E. Punta, L. Fridman, G. Bartolini and S. Delprat, "Nested backward compensation of unmatched perturbations via HOSM observation," Journal of the Franklin Institute, 351, pp.2397-2410, 2014

[24] A. Ferreira, J. Cieslak, D. Henry, A. Zolghadri and L. Fridman, "Output tracking of systems subjected to perturbations and a class of actuator faults based on HOSM observation and identification," Automatica, 59, pp.200-205, 2015.

[25] J. Davila, J. Cieslak, D. Henry, A. Zolghadri and F. J. Bejarano, "A fault tolerant controller based on quasi-continuous high-order sliding mode technique," American Control Conference, 2016.

[26] A. Zolghadri, J. Cieslak, D. Efimov, D. Henry, P. Goupil, R. Dayre, A. Gheorghe and H. Leberre, "Signal and model-based fault detection for aircraft systems", IFAC-PapersOnLine, 48, pp. 1096-1101, 2015.

[27] J. Chen and R. J. Patton, Robust model-based fault diagnosis for dynamic systems. Kluwer academic publishers, 1999. 